Archives de sciences sociales des religions

176 | octobre-décembre 2016

Bulletin Bibliographique

\title{
Émile Poulat et les enquêtes sociographiques de pratique cultuelle (1956-1970)
}

Olivier Chatelan

\section{(2) OpenEdition \\ Journals}

Édition électronique

URL : http://journals.openedition.org/assr/28116

DOI : 10.4000/assr.28116

ISSN : $1777-5825$

Éditeur

Éditions de l'EHESS

Édition imprimée

Date de publication : 31 décembre 2016

Pagination : $55-72$

ISSN : 0335-5985

\section{Référence électronique}

Olivier Chatelan, «Émile Poulat et les enquêtes sociographiques de pratique cultuelle (1956-1970) »,

Archives de sciences sociales des religions [En ligne], 176 | octobre-décembre 2016, mis en ligne le 01 janvier 2019, consulté le 04 janvier 2020. URL : http://journals.openedition.org/assr/28116 ; DOI :

$10.4000 /$ assr. 28116 


\section{Olivier Chatelan}

\section{Émile Poulat et les enquêtes sociographiques de pratique cultuelle (1956-1970)}

Lorsqu'en 1954 Émile Poulat est recruté au Groupe de sociologie des religions du CNRS, le rythme des enquêtes sociographiques de pratique dominicale bat son plein en France. Initié par l'article-programme de Gabriel Le Bras dans la Revue d'histoire de l'Église de France (Le Bras, 1931), l'engouement pour ces recensements religieux a décuplé depuis que le chanoine Fernand Boulard est devenu le principal animateur de cette aventure intellectuelle. En ce milieu des années 1950, ce sont les villes, et en particulier les grandes agglomérations (Marseille et Saint-Étienne en 1953, Lyon-Villeurbanne et Paris en 1954, Toulouse et Bordeaux en 1957) qui retiennent l'attention des enquêteurs parmi lesquels les ecclésiastiques, formés ou non, constituent le gros des troupes. La mesure de l'assistance au culte, affinée grâce à un questionnaire désormais opératoire après les tâtonnements des débuts, est donc le fondement de cette sociographie, descriptive par définition. Une quinzaine d'années plus tard, en 1970, les Archives de sociologie des religions accueillent une recension sévère de Poulat à propos de la synthèse Pratique religieuse urbaine et régions culturelles publiée conjointement par le chanoine Boulard et le sociologue belge Jean Rémy, auxquels la revue donne un droit de réponse inclus dans le même numéro (Poulat, 1970 ; Boulard et Rémy, 1970).

Cette passe d'armes a déjà été repérée par plusieurs historiens du religieux. Claude Langlois l'envisage comme un des moments de la rencontre de la ville par les catholiques français, en reprenant le titre d'un article de Poulat lui-même (Langlois, 2004). Guillaume Cuchet relit cet épisode à la lumière d'une prise de conscience - encore embryonnaire à cette date chez Boulard - d'une rupture dans la pratique religieuse au milieu des années 1960 (Cuchet, 2013 : 107-108). Christian Sorrel livre quant à lui une analyse de ce débat en le resituant dans la trajectoire intellectuelle et pastorale du chanoine (Sorrel, 2013 : 32-36). Enfin, Alain Chenu a proposé une très belle contextualisation d'ensemble de ces enquêtes (Chenu, 2011). On souhaiterait ici apporter une nouvelle contribution en restituant de façon contextualisée les principaux arguments échangés de part et d'autre pour montrer que cette joute constitue le point d'arrivée d'un débat 
qui couve depuis plusieurs années et que l'on peut situer au croisement de plusieurs niveaux de lecture. Il constitue d'abord une étape dans l'élaboration d'une "science catholique » depuis le XIX ${ }^{\mathrm{e}}$ siècle au moins : en négatif des critiques de Poulat se dessine en effet la question des conditions dans lesquelles peut se construire, à la lumière des encycliques et de la tradition de l'Église, un corpus de connaissances, voire une méthode qui soient à la fois orthodoxes ad intra et audibles ad extra. À l'inverse, de façon plus classique, cet échange de vues de 1970 renvoie directement à l'histoire de la construction de la sociologie des religions en France, pensée comme discipline déconfessionnalisée et non limitée à l'étude du seul catholicisme. Le projet fondateur du GSR trouve là un jalon de premier ordre, à la fois parce qu'un de ses membres les plus actifs réaffirme avec force, si l'en était besoin, l'ambition d'une sociologie dégagée de toute instrumentalisation pastorale, et parce qu'elle ouvre une réévaluation du paradigme de la perte religieuse qui dominait alors cette branche des sciences sociales. Enfin, en reprenant l'intuition de Christian Sorrel citée plus haut, on peut aussi envisager ce débat comme un moment commun dans la trajectoire individuelle de deux éminents sociologues du catholicisme, qui est également le combat de deux "géants " dans leurs sphères respectives, lesquelles s'ignorent habituellement : à ma droite un expert du Concile, homme de l'institution et proche de l'épiscopat français (qui apporte dans une large mesure une caution à ses enquêtes), fidèle à sa vocation de prêtre, discret, mais efficace animateur de l'aventure cléricouniversitaire souhaitée par Gabriel Le Bras; à ma gauche, un compagnon de route des prêtres ouvriers devenu chercheur fonctionnaire, acteur de l'institutionnalisation de la sociologie des religions en France et déjà auteur de nombreux ouvrages qui lui offrent une reconnaissance dans le monde scientifique sans commune mesure avec celle du chanoine Boulard dont l'audience reste confinée aux milieux d'Église. Si la postérité de la discipline sociologique donne raison à Poulat, s'attarder sur cette querelle permet de ne pas trop anticiper sur cette victoire et sur le dépérissement annoncé des enquêtes de pratique. C'est en tout cas l'ambition modeste de cette brève étude, qui envisagera d'abord le rapport de Poulat à l'enquête de pratique cultuelle avant 1970 pour aborder avec plus d'appuis, dans un second temps, le débat lui-même.

\section{Émile Poulat et les enquêtes de pratique religieuse avant 1970}

À notre connaissance, Poulat n'a ni dirigé ni participé à aucune enquête de pratique religieuse de type Boulard, de près ou de loin. Comme chercheur, il n'en est pas moins attentif à ce gigantesque effort de recueil des données que son «patron " Gabriel Le Bras avait appelé de ses vœux. Son intérêt prend la forme d'une triple contribution : une série d'études sur la préhistoire des statistiques religieuses en France; des comptes rendus dans le Bulletin bibliographique des Archives; enfin une synthèse publiée en deux articles sur la ville comme fait urbain porteur d'une nouvelle civilisation. 


\section{Écrire une préhistoire des statistiques religieuses}

Entre 1956 et 1960, Poulat entreprend trois études, toutes publiées dans les Archives, à propos d'enquêtes menées en France au XIX et au début du XX ${ }^{\mathrm{e}}$ siècle sur l'appartenance religieuse. L'originalité de cette recherche tient dans la provenance des sources mobilisées : le sociologue utilise des documents extérieurs à l'autorité religieuse, de façon directe ou indirecte.

Le premier article, qui trouve sa place dès le second numéro de la revue en 1956, entend faire le point sur l'existence et, le cas échéant, sur la fiabilité, des statistiques officielles sur les cultes en France (Poulat, 1956b). C'est la première publication de Poulat sur la question des enquêtes au moins partiellement religieuses. Partant du constat que " plusieurs fois au cours du XIX ${ }^{\mathrm{e}}$ siècle, le gouvernement français a réservé une rubrique aux cultes à l'occasion de ses périodiques recensements démographiques ", l'auteur passe en revue la totalité de ces recensements entre 1841 - où apparaissent pour la première fois séminaires et communautés religieuses - et 1872, date après laquelle la III ${ }^{\mathrm{e}}$ République ne prévoira plus de rubrique à caractère religieux. Son but est de connaître l'intitulé des questions et des catégorisations portant sur l'appartenance religieuse des populations dénombrées. Que retient l'auteur de cette chasse à la variable confessionnelle dans ces statistiques de l'âge concordataire ? Essentiellement trois réflexions sur la pertinence de ce type de données. Il en déduit d'abord que la succincte généalogie qu'il a établie donne la possibilité d'étudier les transformations religieuses à l'œuvre, une perspective aussitôt saluée par Gabriel Le Bras dans le même numéro ${ }^{1}$. Le deuxième enseignement est d'ordre technique, mais on verra qu'il constitue de façon récurrente l'hygiène nécessaire du chercheur aux yeux de Poulat: les données sont rendues difficilement exploitables par leur faible homogénéité. Cependant, et c'est le troisième point, Poulat ne cache pas sa surprise de constater que les chiffres exhumés de ces recensements recoupent ceux fournis par le chanoine Boulard - pour la première fois cités - dans ses Premiers itinéraires en sociologie religieuse (Boulard, 1954 : 24) : alors que 97,5\% des recensés se déclarent catholiques à la fin du siècle, 94 \% des Français sont baptisés dans la religion catholique un gros demi-siècle plus tard. Conclusion de Poulat: en dépit "des différences de questionnaire, de la destruction partielle des archives et de l'infléchissement d'une partie des réponses, la déclaration d'appartenance, fondée sur l'ascendance, ne serait pas purement idéale, symbolique ou opportuniste » et rend ces recensements utiles.

La deuxième étude de Poulat prolonge les premiers résultats en effectuant une plongée dans un recensement à l'échelle départementale d'une autre nature : il s'agit de rendre compte d'une enquête anticléricale de pratique religieuse effectuée en 1903 en Seine-et-Marne et publiée dans un journal local, Le Briard

1. "Bref, Émile Poulat nous rend sensible cette évidence que presque toute l'histoire de la statistique religieuse en France reste à écrire » (Le Bras, 1956 : 18). 
(Poulat, 1958a). Les données fournies par cette enquête réalisée au moment de la Séparation importent peu ici. L'essentiel est ailleurs, dans la minutie avec laquelle l'auteur restitue les conditions pratiques de l'enquête, apprécie la représentativité des chiffres et recalcule pour partie les résultats auxquels croient parvenir les rédacteurs du journal. Pourtant, là encore, les données pour Poulat sont non pas secondaires, mais sans valeur intrinsèque : c'est sur la réflexion sociologique qu'elles suscitent, davantage que sur l'information sociographique qu'elles révèlent, que le chercheur doit s'attarder. Il évoque au passage quelques pistes suggestives pour ceux de ses lecteurs qui pensent, comme lui, que «toute une préhistoire de la sociographie du catholicisme serait à faire " : Hippolyte Taine, dès 1891 dans la Revue des Deux Mondes, et Anatole France, dans L'Église et la République en 1904, ont perçu qu' «il y a plusieurs raisons d'aller à la messe, qui ne sont pas toutes des raisons pieuses » (Ibidem:128).

Enfin, c'est à partir d'une enquête socialiste internationale menée en 19021903 que Poulat reprend le dossier des statistiques sur le culte (Poulat, 1960a). Dans la première note de bas de page de cette longue étude dans laquelle l'auteur laisse largement la parole aux acteurs pour faire entendre " un dialogue à plusieurs voix ", l'auteur revient sur ce qui s'apparente à un trou de mémoire: "Aucun inventaire n'a encore été fait de ces enquêtes ", déplore-t-il, avant d'indiquer une dizaine d'exemples de ces sources possibles, pour la plupart dispersées dans des revues du début du siècle (Ibidem: 109). Cette recherche est à mettre en lien avec la préparation de sa grande introduction au Journal d'un prêtre de l'après-demain de l'abbé Calippe que publie Poulat l'année suivante (Poulat, 1961). Un bilan intermédiaire s'impose à la lecture de ce premier dossier. Une question revient sous la plume de l'auteur dans les trois articles cités : que vaut cette enquête ? Quelle valeur accorder à ses résultats ? Ce positionnement critique, qui le conduit à reprendre les calculs lorsque les données sont encore accessibles, est une marque de fabrique : si l'attention portée à la fiabilité des informations de première main est si grande, c'est qu'elle constitue le soubassement de l'analyse en dehors duquel toute interprétation est inévitablement vouée à l'erreur. Cette passion du chiffre est donc plutôt inquiétude. Sa curiosité pour les recensements anciens est par ailleurs une preuve de l'intérêt indirect que porte Poulat aux enquêtes alors en cours sous l'impulsion du chanoine Boulard : chacun creuse son sillon, à distance, l'un par la correspondance avec le clergé local des paroisses de France qui fournit données et diagrammes, l'autre par " ce long commerce avec les documents » (Groupe de sociologie des religions, 1969:44) qui fait de Poulat un historien plutôt qu'un sociologue, soucieux de la longue durée et de la sédimentation des savoirs.

\section{Le Bulletin bibliographique : une veille sans complaisance}

Le « BB » est le lieu des Archives. C'est à la fois le réceptacle de toutes les publications dans le champ de la sociologie des religions et un espace 
d'expression apprécié des recenseurs. Poulat y fait là son nid, s'il est vrai que cette rubrique lui permet de concilier son "appétit féroce en matière bibliographique ", un «dispositif formateur pour l'équipe initiale » et une "forme privilégiée d'invention et d'expression pour les idées les plus audacieuses » (Maître, 2001 : 27). C'est par conséquent aussi dans le Bulletin qu'il faut repérer un éventuel intérêt de Poulat pour les enquêtes de pratique cultuelle. Signalons d'emblée que ce n'est pas lui qui assure la majorité des comptes rendus pour les publications en lien avec cette question. Il n'est pas considéré au sein du GSR comme le spécialiste de ces enquêtes, ce qui correspond à une réalité : ses recherches ne portent pas directement sur ce champ. Dans le Bulletin, ce sont principalement François-André Isambert et Jacques Maître qui rendent compte de l'état de la recherche sur les enquêtes Boulard. Le premier s'est occupé en 1952 d'un recensement dominical à l'échelle d'un arrondissement parisien et est l'auteur, avant sa thèse principale sur Buchez publiée en 1967, d'une dizaine d'articles sur les méthodes et les résultats des enquêtes sociographiques ${ }^{2}$. Le second tient à jour le tableau des recensements religieux, dont il publie les résultats dans les Archives ${ }^{3}$.

Au total, pour la période 1956-1970, Poulat signe pour le Bulletin six recensions portant sur des enquêtes ou sur la "sociologie pastorale" au sens large. Elles sont de taille variable, de douze lignes pour La pratique dominicale. Carte religieuse $d u$ diocèse de Nice d'E. Diebold (1955), première de la série, à une colonne et demie pour L'équipement paroissial d'un diocèse urbain : Paris, 18021956 (1957) d'Yvan Daniel ${ }^{4}$. À l'exception, notable, de ce dernier ouvrage pour lequel le sociologue salue "l'austérité » et la «minutie » du travail fourni, tous ces comptes rendus se montrent particulièrement critiques, selon un schéma assez répétitif. Après avoir souligné le travail consciencieux de l'enquêteur, le propos en vient rapidement à souligner les faiblesses méthodologiques : insuffisances du questionnaire, manque de différenciation des informations recueillies, absence de croisement avec d'autres données. C'est le cas en particulier pour l'étude du sociologue bruxellois Michel Faerman sur la pratique religieuse à Mont Saint-Guibert ou celle du P. Jesus-Maria Vasquez sur un quartier madrilène publiée en $1958^{5}$. Poulat se méfie également des explications rapides ou trop générales. Il en appelle quasiment systématiquement à davantage de rigueur chez ses collègues, dans le traitement de l'information comme dans l'interprétation des résultats.

Deux essais subissent tout particulièrement le feu de la critique. Celui du prêtre strasbourgeois Paul Winninger d'abord, dont le Construire des églises

2. «François-André Isambert. Éléments biographiques », consultable à l'adresse : <assr. revues.org/25703>.

3. Voir en particulier Maître, 1957.

4. Voir Poulat, 1956a et Poulat, 1957a.

5. Voir Poulat, 1957b et Poulat, 1958b. 
(1957) est pourtant un best-seller dans les diocèses de France. Après en avoir rappelé la thèse - multiplier les lieux de culte pour rendre plus efficace un apostolat urbain "à échelle humaine " - Poulat épingle plusieurs affirmations glanées au fil des pages pour en souligner la faiblesse argumentative : "Est-il bien établi que "l'absence de paroisses est une des causes principales de la déchristianisation des centres urbains au XIX siècle” (p. 44) ? Que "le progrès social, en réduisant peu à peu la misère matérielle et la révolte morale qui l'accompagne souvent, rend les âmes plus disponibles" (p. 62) ? [...]. Que "sociologiquement la notion de classe est de moins en moins valable" et que "l'opposition brutale des classes sociales est heureusement en déclin” (p. 71) ? (Poulat, 1957c : 207). L’usage du doute méthodologique est pour Poulat une façon de "s'équiper " pour l'exploration disciplinée d'un phénomène : il y a là l'expression d'une ascèse intellectuelle qui refroidit les enthousiasmes les moins naifs. L'autre victime du censeur est le Manuel d'initiation à la sociologie religieuse publié par Joseph Laloux en 1967. Outre une condamnation sans appel de « la sociologie religieuse comme science auxiliaire de la pastorale » revendiquée et développée par l'auteur dans toute la première partie - «cent pages pour tant de choses, c'est peu, suffisant toutefois pour illustrer la confusion initiale »-, l'absence des auteurs classiques de la sociologie, dont Le Bras, est perçue par Poulat comme « une injustice ", pis, comme une faute : "Et puisque ce livre, nous dit-on, vise avant tout le clergé, est-ce le bien servir que l'entretenir ainsi dans ses errements et le contenir dans l'enceinte mentale dont précisément il éprouve le besoin et proclame la nécessité de sortir ? Il est vrai que, dans cette perspective, une telle question ne relève pas de la sociologie, mais de la pastorale» (Poulat, 1967). Les critiques vigoureuses qu'exprime Poulat à propos du livre de Boulard et Rémy en 1970 ne sont donc pas neuves : on les trouve déjà, dispersées au gré des comptes rendus, dans le Bulletin. Il en va de même pour l'interprétation que fait le chercheur de l'urbanisation et de sa "découverte " par les catholiques: Poulat a écrit deux articles sur ce sujet en 1960-1961 qui fourniront dix ans plus tard le canevas de son argumentation.

\section{L'urbanisation, une variable de la pratique religieuse?}

Le double article, l'un paru dans les Annales dans le numéro de novembredécembre 1960 (Poulat, 1960b), l'autre dans les Cahiers internationaux de sociologie en janvier-juin 1961 (Poulat, 1961), occupe une position originale dans la production éditoriale catholique consacrée à la ville et à l'urbanisation. Il fait suite à une grande série de contributions concentrées autour des années 19531956 qui révèlent les premiers résultats des enquêtes sociographiques dans les paroisses urbaines. Ainsi, la livraison de février 1955 de la Chronique sociale de France ouvre ses colonnes à plusieurs spécialistes de sociologie religieuse urbaine, parmi lesquels Denis Szabo, Jean Chélini, François Houtart, Norbert Lacoste, Lucien Gros ou Maurice Blanc de la Fontaine. Suivront au sein de la même 
revue en 1955-1956 des mises au point techniques de sociologues lyonnais (Jean Labbens, Émile Pin, Roger Daille) avant que les Études de sociologie religieuse (1956) de Gabriel Le Bras viennent compléter les Premiers itinéraires en sociologie religieuse du chanoine Boulard, première tentative de synthèse sur la question publiée deux ans auparavant. Inversement, le double article de Poulat précède une seconde vague de publications au cours de l'année 1965, avec des dossiers portant sur la ville dans trois revues (La Nef, Économie et Humanisme et Citoyens 60) et une Semaine sociale de Brest consacrée à cette question. Dans cet entre-deux, Poulat affirme que la ville est devenue un fait majeur qui s'impose désormais à la conscience des catholiques de France.

On pourrait donc s'attendre logiquement à ce que l'auteur fasse des enquêtes de pratique dominicale dans les agglomérations le point de départ de cette " découverte ", selon ses propres termes. Poulat, comme souvent, n'est pas là où son lecteur l'attend. Il a fait un pas de côté pour observer, au-delà des résultats des recensements religieux, la " crise » de la paroisse urbaine. Il n'est certes pas le premier à se pencher sur la question : l'onde de choc de La France, pays de mission? des abbés Godin et Daniel en 1943 puis les définitions concurrentes de la paroisse urbaine ont suscité un vif débat après-guerre (Fouilloux, 1997 : 147-160). Mais il en propose une généalogie et une théorie qui, sans le dire, relativisent considérablement la portée des enquêtes sociographiques alors en cours d'exploitation.

Résumons-en les principales idées. Si la paroisse des villes est en crise, c'est parce que la représentation religieuse traditionnelle de l'espace urbain ne tient plus : celui-ci n'est pas un tissu à découper ou à lotir, mais bien le lieu d'une nouvelle civilisation, qui croise multiplicité des groupes et éclatement des lieux dans un contexte de mobilité croissante. Pour faire face à cette situation inédite, la paroisse urbaine s'adapte - en puisant dans un panel de stratégies depuis le « camp retranché » jusqu'au " camp de base »- ou invente des dispositifs autonomes à partir de trois ancrages possibles: la résidence, l'origine ou la profession/milieu. Il n'est question nulle part d'une influence régionale qui viendrait lisser la spécificité de la ville. Pour Poulat, les fonctions religieuses urbaines devraient constituer le nouveau terrain de chasse du chercheur en sociologie des religions.

Ce double article a-t-il été lu par Boulard et Rémy avant la publication de Pratique religieuse urbaine? Si tel est le cas, les deux auteurs ne l'ont visiblement pas intégré dans leur analyse. C'est bien ce que Poulat leur reproche, entre autres critiques. À leur décharge, il faut reconnaître à sa juste valeur l'ouvrage qu'ils publient en 1968 et l'objectif premier de cette entreprise de haut vol : il s'agit ni plus ni moins de synthétiser en 200 pages la masse des informations recueillies depuis une vingtaine d'années pour les ressaisir au travers d'une interprétation qui rende compte d'invariants et de discontinuités. C'est le couronnement d'un projet fou, ce qui peut expliquer que les auteurs n'aient pas, par ailleurs, lu la 
totalité de la littérature disponible sur le sujet. L'inverse en tout cas est avéré : Poulat attendait sur le quai pour ouvrir avec délectation le trésor ramené du large après des années de navigation en haute mer. Il peut désormais en faire l'inventaire critique.

\section{Le débat de 1970 : une leçon magistrale de sociologie}

On peut structurer la discussion autour de quatre enjeux principaux : la nouveauté des conclusions auxquelles parviennent les auteurs de Pratique religieuse urbaine; la mise en évidence de la région comme variable explicative; le rôle historique de l'institution religieuse dans les phénomènes observés ; enfin l'objet même de ce que doit être la sociologie religieuse en 1970.

En recenseur accompli, Poulat commence son compte rendu en replaçant l'ouvrage dans le continuum des études sociographiques de pratique cultuelle: pour la première fois en France, les chercheurs ont à leur disposition des statistiques précises pour l'ensemble des villes françaises, c'est-à-dire toutes celles de plus de 50000 habitants et presque toutes celles de plus de 25000 habitants. C'est, en soi, un événement pour la jeune sociologie religieuse. Le temps est donc venu du traitement général de cette gigantesque masse de données pour dégager une synthèse. Quelle que soit la qualité de celle-ci, elle sera appelée à faire date : " À l'avenir, on ne pourra que repasser derrière ou reprendre le travail à la base » (Poulat, 1970 : 97). Cette responsabilité scientifique des deux auteurs, soulignée dès le début de l'article, existe très en amont, dès le moment de la collecte des données: comme il le fait de façon systématique dans le Bulletin, on l'a vu, Poulat pose la question de la fiabilité du matériau rassemblé. Or, constate-t-il, rien n'est moins sûr concernant les informations brutes collectées : leur hétérogénéité aurait pu ruiner dès le départ toute tentative de croisement des données. Poulat prend acte de l' "effort courageux " et du "positivisme terre à terre " des deux auteurs : un long et fastidieux travail préalable de normalisation des informations a rendu leur comparaison possible. Sans doute aussi l'enjeu principal pour Poulat n'est-il pas seulement dans une querelle de chiffres : poser la question de la qualité des données, mais sans s'y appesantir outre mesure, c'est faire son travail de recenseur tout en se dégageant de l'espace pour en venir à des questions plus fondamentales.

Il faut dire que les conclusions de Boulard et Rémy sont loin d'être anodines. Elles embrassent et résument en quelques sentences la sociologie du catholicisme français de l'après-guerre. Rappelons-les ici pour plus de clarté : la pratique religieuse des ouvriers est nettement inférieure à celle des autres groupes socioprofessionnels; la pratique est très variable selon les villes (de 5,5 à $39 \%$ ), mais avec des écarts beaucoup plus resserrés qu'à la campagne et à une moyenne inférieure au taux national (entre 15 et $20 \%$ selon les bases retenues); il n'existe aucune corrélation avérée entre pratique religieuse et chacune des variables suivantes: 
le pourcentage d'ouvriers dans la population active d'une ville, la place de l'industrie dans une ville, la structure d'âge ou de sexe de la population urbaine, la dimension des villes ou encore leur rythme de développement. Poulat résume : "Ainsi donc, si les villes sont en moyenne générale moins "chrétiennes" que les campagnes, les grandes villes ne sont pas plus "déchristianisantes" que les petites, et les villes les plus ouvrières ou les plus industrielles ne se montrent pas spécialement "déchristianisées". [...] La ville constitue un contexte global qui agit sur l'ensemble de ses sous-populations. En revanche, une corrélation apparaît entre ce contexte et son environnement, entre la ville et sa région : la disparité religieuse entre villes s'enracine dans la diversité régionale de la France. Telle est l'hypothèse autour de laquelle s'est construit le livre: "C'est l'implantation des villes en régions culturelles diverses qui explique fondamentalement la dispersion de leurs taux de pratique" (p. 57) (Ibidem: 99). Mais est-ce là un ensemble de conclusions neuves? Poulat rappelle que la corrélation entre catégorie socioprofessionnelle et pratique religieuse a été établie bien avant la publication du livre, sans préciser cependant de références bibliographiques. Il est effectivement avéré que l'on trouve cette démonstration dans plusieurs ouvrages dès le milieu des années 1950 (Pin, 1956 ; Labbens et Daille, 1957). D’autre part, le recenseur signale que Georges Gurvitch et Maurice Halbwachs ont déjà montré dans leurs travaux que la ville est un phénomène global et non enclos sur lui-même. Bref, remarque Poulat non sans une certaine condescendance, " on est donc là devant deux thèses classiques de la sociologie française ».

Fernand Boulard répond par deux arguments, l'un scientifique, l'autre pédagogique. Dans un paragraphe subtilement intitulé "Les acquis d'une discussion critique ", le chanoine ne se prive pas de constater que les attaques répétées de Poulat contre le traitement statistique mis en œuvre dans Pratique religieuse urbaine ne font que renforcer la validité des conclusions apportées par les deux auteurs : "Nous constatons que 15 pages de critiques minutieuses, voire pointilleuses, ont laissé inentamée notre démonstration fondamentale : la dispersion des taux globaux de messés dans les villes françaises ainsi que celle des taux de pratique de leur population ouvrière ne se trouve en corrélation positive qu'avec une seule variable : le niveau global de pratique de leur environnement régional. Aucune des variables habituellement alléguées ne fournit de corrélation acceptable » (Boulard, Rémy, $1970: 117-118$ ). Face au soupçon de "déjà vu »-ou déjà lu - émis par Poulat, Boulard rappelle que son livre ne s'adresse pas qu'aux sociologues au fait des dernières avancées, mais à un auditoire plus commun, que lui pratique plus souvent que son interlocuteur : « Nous trouvons É. Poulat quelque peu optimiste : notre expérience de publics fort divers nous a convaincus que la démonstration était à faire " (Ibidem : 121). Des réponses courtoises, mais fermes : la première passe d'armes ne procure aucun avantage comparatif, mais elle dessine déjà la ligne de défense de Boulard. À l'hypercriticisme du chercheur du GSR, le coordinateur des enquêtes oppose le bon sens du travail bien fait : la démonstration tient toujours; il n'est jamais inutile de répéter pour convaincre. 


\section{La région, explication ultime ou simple intuition?}

Le débat porte sur la définition du fait urbain. Comme on l'a vu, Poulat a déjà étudié la question dans des articles antérieurs à 1970. On se contentera donc ici de donner ses critiques nouvelles. Primo, le périmètre statistique retenu par Boulard et Rémy oscille sans cesse entre la ville et l'agglomération, selon les informations recueillies localement. Ce "flottement " laisse en outre de côté les banlieues, "qui font ici figure de parents pauvres ». Secundo, en introduisant ce flou dans la délimitation topographique de ce qui fait la ville, l'absence de corrélation entre la taille des villes et le niveau de pratique religieuse peut s'avérer une erreur. La réponse de Boulard consiste en une nouvelle série de démonstrations mathématiques à partir de deux nouveaux tableaux statistiques construits par le chanoine ${ }^{6}$. La discussion roule sur la question, déterminante, des seuils et des catégories. Le chanoine conclut au terme d'une analyse serrée de deux pages : «Et l'on a envie de retourner à son auteur sa réflexion terminale : "Argumentation et découpage seraient-ils donc frères siamois?” "(Boulard, Rémy, 1970 : 120).

C'est le même enjeu du découpage qui introduit le cœur de la critique de Poulat sur la région comme variable explicative. Si le Bassin parisien est une unité retenue par Boulard et Rémy pour justifier des différences de pratique à l'intérieur et à l'extérieur, « comment donc [le] délimiter ? Les auteurs n'en disent rien et on ne voit pas leurs critères ". À cette première difficulté s'en ajoute une seconde, plus redoutable, qui porte sur le type de raisonnement. Si la ville, comme l'affirme Pratique religieuse urbaine, est interprétée comme un phénomène total agissant comme une sous-culture dans la culture régionale englobante, " on change de terrain » dit Poulat:

Qu'est-ce, en effet, que cette " variable explicative » et qu'a-t-elle la vertu d'expliquer ? Et est-ce donc sans raison si, brusquement, le style et la méthode changent aussi ? Une corrélation, jusqu'alors négligée dans ce genre de travaux, s'est imposée, et seule, tandis que toutes les autres, rendues pourtant si familières, refusaient d'apparaître. C'est une question; ce peut être le départ d'une explication; ce n'est en rien une explication. La technique qui a permis de la dégager est impuissante à éclairer sa signification. Il faut ou s'arrêter, ou faire un saut, mettre en jeu d'autres ressources. Les auteurs ont choisi de poursuivre : on les en louera, même si l'on accueille avec plusieurs réserves le double mouvement qu'ils opèrent à partir de la base établie.

6. Fernand Boulard justifie systématiquement le choix de ses variables. Ainsi à propos de la liste des 105 villes ou agglomérations dont Poulat doute du bien-fondé : «Pour l'établissement du tableau général, nous avons pris comme critères le territoire urbain réellement recensé par l'enquête religieuse, et son volume de population au moment de l'enquête : c'est pourquoi nous avons éliminé un certain nombre de villes ou agglomérations qui avaient pourtant plus de 25000 habitants en 1962, pour la raison qu'elles ne les atteignaient pas lors de l'enquête dominicale, soit à cause de sa date (tout un groupe est autour de 1954), soit à cause d'un territoire recensé plus exigu »(Boulard et Rémy, $1970: 124-125$ ). 


\section{Quelques paragraphes plus loin, Poulat conclut :}

L'évidence s'est imposée: il n'y a pas de déterminisme factoriel simple, mais une totalité englobante. N'étant pas simple totalité de facteurs endogènes, cette totalité englobante est à son tour et en même temps une totalité englobée : ce que manifeste la solidarité régionale. On pourrait continuer, car la région est, elle aussi, une totalité englobée [...]. À ce point, on a situé, mesuré, ordonné des variables ; on n'a pas expliqué le jeu (Poulat, 1970: 100 et 108).

Le chercheur du GSR peut décocher alors une troisième série de flèches sur la définition même de la région culturelle que les deux auteurs considèrent comme l'explication ultime des taux différenciés de pratique cultuelle. Après avoir longuement expliqué ce qu'il faut entendre par "écologie sociale » à partir de la notion de milieu et restitué le parcours historiographique du mot " région " en le tenant de bonne source - les travaux récents du géographe Paul Claval -, Poulat donne une leçon de sémantique à qui veut l'entendre :

Tout le trousseau des variables a donc été consciencieusement essayé. Une clé s'en est dégagée. Elle a nom région. Souvent répété, le mot n'est nulle part défini, précisé, analysé. Ce n'est d'ailleurs pas un mot, mais une famille : "région culturelle », "région socio-culturelle ", "région géographico-culturelle ", " aire géographico-culturelle ", y sont tenus pour équivalents et interchangeables. [...]. On y surprend un mélange franco-franglais, l'histoire de France passée au langage de l'anthropologie anglosaxonne. De la culture, qui se caractérisait comme une idéologie de l'élite et une utopie de l'universel, on glisse à des cultures, qui se chargent de tout ce qui sépare l'homme de la nature, mais, surtout, qui se présentent comme autant de visages opposés à la volonté centralisatrice par le particularisme de terroir. Visages voilés : cette culture régionalisée reste une forme vide, une allusive référence théorique, appelée à unifier deux champs séparés d'observation, les unes numériques (traitées par analyse secondaire, mais de première main) et les autres historiques. Sur cette culture, l'ouvrage n'a pas une ligne (et ce n'est certes pas ignorance) (Ibidem : 106).

Poulat se met alors à la recherche de ce qui, dans Pratique religieuse urbaine, semble caractériser une région au sens où l'emploient les auteurs. Il en tire deux constats. D’une part, la région de Boulard et Rémy est une formation complexe, qui recouvre l'ensemble des déterminations sociales, démographiques ou économiques, en tension avec la ville et nourri de processus de longue durée. Dans cette inertie, Poulat croit déceler ce qui, au fond, intéresserait les deux auteurs dans leur insistance sur le fait régional : une "résistance culturelle à l'agression extérieure ", qu'il rapproche des contrastes séculaires régionaux mis en évidence, " depuis longtemps déjà », par le doyen Le Bras. D’autre part, la région repose sur l'affirmation que ville et campagne environnante ont plus de points communs que de différences : la symbiose l'emporte sur les kystes, pour reprendre le vocabulaire du livre. Dès lors, si la région culturelle n'est définitivement pas, pour Poulat, " une théorie explicative ", " on peut y voir ici, en revanche, l'intuition unificatrice d'un champ problématique. Ce n'est pas négligeable, et volontiers on en prendra acte" (Ibidem : 107). Boulard voit dans cette concession un "point d'accord fondamental». 
Pour le reste, la réponse du chanoine sur la définition de la région n'est pas totalement convaincante. Dans un paragraphe intitulé "Quelques débats plus fondamentaux » - qui fait suite à une explication très détaillée de l'élaboration théorique de la liste des 105 villes sur laquelle il fonde son analyse -, il reconnaît que la notion de " région culturelle » est un élément central du livre qui n'a pas été suffisamment explicité. Boulard convoque d'abord Les espaces économiques, une brève introduction parue en 1961 dans la collection "Que sais-je ? ». Son auteur, Jacques Boudeville, est un économiste de l'école de François Perroux, qui mobilise une conception dynamique de l'espace pour penser la région des géographes. De fait, le sociologue distingue "régions homogènes » et " régions polarisées », mais ses explications se font moins claires lorsqu'il aborde le problème particulier de la région culturelle :

Elle [la région culturelle] se présente comme une entité spécifique, dans la mesure où elle est une zone homogène dont la constitution dépend en partie d'une polarisation. On la découvre comme une zone qui présente des caractéristiques internes dissemblables des caractéristiques externes. Il peut s'agir de la délimitation des divers patois, de zones où prédomine tel type de jeu populaire... D'après les types et nombres d'indices que l'on superpose, on peut aboutir à des délimitations différentes. Lorsqu'on s'interroge sur la genèse de ces caractéristiques communes, elles apparaissent comme des solutions progressivement trouvées à des problèmes posés à la vie sociale (Boulard, Rémy, 1970 : 126-127).

Mélange d'historicisme et d'oppositions de contraires un peu schématiques, vocabulaire relativement flou : on pourrait croire Boulard peu à l'aise avec ces notions. Pourtant, à lire de près son analyse, on voit poindre entre les lignes un espace-type qu'il connaît bien pour l'avoir inlassablement proposé comme grille de lecture auprès des évêques de France : la "zone humaine ", fondement de la pastorale d'ensemble des années 1960. Carrefour d'influences, ancrée dans un territoire (à la différence de l'Action catholique) qui présente des problèmes communs de vie sociale et d'Église, cette «zone humaine » est au cœur du dispositif pastoral souhaité par l'épiscopat au moment du Concile. Néanmoins, Boulard n'en parle pas : c'est l'assise théorique de la région culturelle qui doit être ici fortifiée, non ses développements d'ordre institutionnel. Mais le chanoine éprouve une certaine difficulté à justifier scientifiquement ce découpage par régions culturelles. Il pense s'en extraire en décomposant ces régions culturelles à l'aide de trois " critères dialectiques " : les facteurs à impact global ou partiel ; les facteurs dont l'effet est lié à la proximité spatiale de la population ou qui au contraire en sont relativement indépendants ; enfin les facteurs d'origine ancienne ou bien récente. S'en suit un exposé des différents résultats selon les combinaisons de ces variables qui aboutit à une conclusion qui était en quelque sorte posée d'avance avec ce choix de critères : "Le centre de l'analyse consiste à refuser une explication unifactorielle [...]. La région continue à apparaître comme un facteur à action globale, c'est-à-dire agissant de façon homogène sur les diverses catégories de population. La région culturelle n'est pas anéantie comme variable explicative. Son interrelation aux autres variables reste la même » (Ibidem :130). 
Fernand Boulard définit finalement cet espace régional comme le réceptacle des héritages culturels.

\section{D'où viennent ces invariants culturels régionaux ?}

Boulard et Rémy s'efforcent de comprendre les raisons pour lesquelles la diversité régionale est en corrélation directe avec la diversité de la pratique culturelle. C'est là qu'intervient l'appel à l'histoire ou plutôt à la sociologie historique, qui porte avec elle le temps long, gage de profondeur dans l'interprétation des résultats. Le chanoine s'appuie sur des références inattendues pour étayer sa démonstration :

Comme la plupart des héritages culturels, la famille est agent privilégié de transmission. Par là, nous rejoignons les thèses de Pierre Bourdieu relatives à l'incapacité où se trouve l'école de neutraliser les différences liées à l'héritage culturel familial, surtout lorsqu'il s'agit de transmettre une familiarité par rapport à des codes non techniques. La transmission de ces éléments, importants pour induire nos réactions les plus réactives et les plus quotidiennes, se fait à travers des processus non conscients. Et nous faisons l'hypothèse qu'il en est ainsi pour les éléments qui nous apparaissent comme les plus intimes au plan de la conscience religieuse (Ibidem : 132).

Ce deus ex machina bourdieusien permet au chanoine de faire de l'institution religieuse le facteur explicatif en dernière analyse. Pratique religieuse urbaine l'affirme et Boulard le répète à son recenseur : le facteur le plus explicatif des diversités religieuses de 1970 est interne au christianisme et il est à chercher dans l'histoire de la pastoration.

Poulat retrouve là un terrain qu'il connaît bien et il peut tout à loisir relever les insuffisances de la démonstration des deux auteurs. Il dénonce d'abord une " histoire panoramique ", c'est-à-dire allégée de dates et des rapports complexes entre les différents "secteurs" (économique, social, religieux, etc.) des sociétés humaines. Ensuite, il décèle chez les deux auteurs une contradiction dans le propos. Si l'action du clergé porte en elle-même la responsabilité de ses échecs et de ses réussites, alors, comme ils l'écrivent, "le dynamisme de l'institution religieuse se traduit par sa capacité d'acculturation» (p. 88). Il a lors beau jeu de renverser les conclusions de ses interlocuteurs : "Ça n'a l'air de rien, mais, si la formule n'est pas vaine - encore qu'elle soit vague -, le morceau est énorme, et à plusieurs titres que je négligerai, sauf un. Car faire de l'acculturation l'exigence, et donc, en un certain sens nécessairement, la norme de la pastoration, n'est-ce pas rendre leur primauté aux “facteurs externes" ? Qu'il s'agisse pour l'Église de s'acculturer elle-même au monde ou de s'acculturer le monde à ellemême, l'acculturation est d'abord réponse à un stimulus extérieur, à une initiative étrangère » (Poulat, 1970 : 110). Boulard et Rémy marquent leur accord avec Poulat sur la nécessité de s'interroger sur la réponse, diverse, donnée à la proposition de la foi, de son acceptation pleine et entière jusqu'à son refus radical : la question du « dialogue culturel entre l'évangélisateur et le peuple récepteur » est un enjeu que Boulard admet volontiers. Poulat pour sa part voit dans 
cette recherche historique sur les origines d'une différenciation de la pratique la quête d'un « secret perdu ", celui de la recette d'une acculturation réussie dans certaines régions. Il s'agit là d'un point crucial, car il dessine en creux des conceptions différentes de ce que doit être la sociologie religieuse de 1970.

\section{Le « mur » ou les deux conceptions de la sociologie religieuse}

En faisant de l'action pastorale l'élément explicatif, in fine, de la diversité française en matière de pratique cultuelle, Boulard et Rémy retrouvent au bout du chemin le sens même de leur vocation de sociologues et la justification de plusieurs décennies de labeur au service des enquêtes : pastorale et sociologie se soutiennent mutuellement. Pour le chanoine, la relation fonctionne dans les deux sens. La pastorale est un objet de la sociologie. C'est ce qu'il enseigne depuis plusieurs années à l'Institut catholique de Paris : l'organisation sociale de l'Église, ses modèles apostoliques et plus largement la culture religieuse peuvent se prêter à une grille de lecture de sciences sociales. Mais la sociologie peut aussi participer à l'élaboration d'une stratégie pastorale. À condition, précise Boulard, de bien délimiter les rôles. Le chercheur est un analyste, pas un conseiller. Cette assignation se justifie doublement : l'autorité religieuse reste maître de sa politique et seule compétente en matière de normes de conduite; le sociologue n'est pas toujours apte à comprendre "du dedans ", et les hommes et les choses (Boulard, Rémy, 1970 : 137). De toute façon, affirme Boulard à la fin de sa réponse, «il serait illusoire de rêver d'une concorde préétablie » entre le sociologue et l'homme d'action, comme pour mieux souligner ce qui le sépare de son homologue au Groupe de sociologie des religions.

Dans sa recension, Poulat va, quant à lui, bien au-delà de la distinction entre sociologie «pure » et sociologie « appliquée ». Il déplace le questionnement en affirmant que la recherche de causalité engagée par Rémy et Boulard conduit immanquablement à une impasse de la pensée : en dépit de tout le matériau accumulé et traité depuis des années, il est illusoire de croire que seule une meilleure adaptation de la pastorale aurait permis une christianisation plus durable dans certaines régions et aurait donc, corrélativement, freiné la déchristianisation. Car, affirme Poulat, c'est bien de déchristianisation dont il s'agit : le vaste mouvement des enquêtes enclenché par Le Bras en 1931 a, chemin faisant, progressivement perdu de vue son objet initial - la conformité d'un peuple aux préceptes canoniques et moraux grâce à des indicateurs chiffrés - pour enregistrer au contraire un détachement de ce peuple à l'égard de l'Église, dont le sociologue s'évertue à chercher les raisons. "On ne devrait donc pas s'étonner ", assure Poulat, que la recherche plafonne, " comme si elle se heurtait à une sorte de mur sociologique qu'elle ne voit pas comment franchir, qu'elle ne paraît pas toujours soupçonner»(Poulat, $1970: 114$ ). À dire vrai, cette idée a déjà été exprimée par Poulat, dans un article publié dans Social Compass en 1969. Il est surprenant de constater que celui-ci n'a pas hésité à reprendre mot pour mot 
deux pages complètes de cet article pour compléter sa recension du livre de Boulard et Rémy ${ }^{7}$. Ce qui relativise la dimension strictement bilatérale de l'échange de vues entre Boulard et Poulat : celui-ci développe une argumentation qui dépasse le strict cadre de l'exercice canonique et fournit un véritable programme, de son aveu même ${ }^{8}$. Quel est ce programme assigné à la sociologie ? Il faut, dit Poulat, ne pas opposer la mesure d'une pratique - aisément accessible par le chiffre et qui dépendrait seulement des fidèles - à la foi - qui relèverait du magistère seul et difficilement quantifiable. Car la première est aussi fondamentalement l'expression de la seconde. À quoi croient les croyants ? Qui prientils ? Poulat ne pense pas que le religieux soit immuable, en quoi il revendique une différence avec la conception lebrasienne ${ }^{9}$. Il invite à repenser l'objet de la sociologie religieuse, dans une optique d'analyse de la sécularisation bien que l'auteur se refuse dans la suite du texte à utiliser le mot :

On aura beau, dès lors, manipuler dans tous les sens les variables et les variations de la pratique, en généraliser le concept par l'attention à toutes les formes de vitalité religieuse, jamais on n'en tirera ni le fait capital qui est à expliquer ni même un embryon d'explication de ce fait : pendant de siècles, Dieu, le Surnaturel, le Ciel et l'Enfer, Jésus fils de Dieu, les miracles, le Diable, ont été des présuppositions absolues de l'Europe chrétienne que n'entamaient pas l'aberrance d'esprits forts ou de petits groupes, et aujourd'hui, non seulement d'absolues elles sont devenues litigieuses, mais plus encore, même aux yeux des croyants, elles ont cessé d'apparaître comme le fondement nécessaire de l'ordre politique qui s'est institué et qu'ils acceptent. Le consensus social repose sur d'autres bases, sur un ensemble d'évidences devenues communes, qui s'est progressivement et péniblement constitué, et dont la révélation chrétienne avec son histoire sainte s'est trouvée exclue à mesure. On peut en juger de plusieurs manières, $\mathrm{y}$ voir un progrès ou un recul, et même un progrès de la raison humaine à effets de sens contraires, où la vie religieuse a gagné en qualité ce qu'elle a perdu en nombre. Mais le fait s'impose : le surnaturel est désormais matière à option et à débat, c'est-à-dire affaire de conscience et non plus la loi d'une société ${ }^{10}$.

Fiabilité de l'Église et crédibilité de son enseignement constituent les deux dimensions de ce problème nouveau. Mais cette question-là, Boulard et Rémy pouvaient-ils la formuler en ces termes?

Il faut redire ici la qualité du débat dont les Archives de sociologie des religions se sont fait l'écho. On n'en attendait sans doute pas moins de ses protagonistes, mais encore faut-il le reconnaître pour en prendre la mesure : deux

7. Les pages 114 à 116 (jusqu'à la note 26) correspondent aux pages 476-478 de l'article de 1969.

8. "C'est moins une critique qu'un programme qui vient d'être suggéré. Il s'impose comme la tâche urgente devant le mur évoqué plus haut " (Poulat, 1970 : 116).

9. Il s'en est expliqué dans un remarquable article publié dans la revue Critique (Poulat, $1957 d)$.

10. Ibidem : 115-116. Danièle Hervieu-Léger a également utilisé ce paragraphe pour montrer que Poulat ne s'en tiendrait pas aux théories de la sécularisation, mais proposerait de penser une réarticulation de la culture catholique avec d'autres cultures qui la travaillent et la dépassent (Hervieu-Léger, $2001:$ 87-90). 
articles de plus de 60000 signes chacun, au contenu dense et minutieusement référencé, truffé de calculs et d'explicitation de ces calculs - parfois refaits ou corrigés. Fernand Boulard ne ménage pas sa peine pour contrer les assauts de son lecteur-recenseur : certes, il a l'avantage de clore la discussion puisque la rédaction des Archives lui a précisé au préalable que le dernier mot lui serait laissé. Mais il est incontestablement à la hauteur de l'échange de vues, sans doute plus courtois et moins provocateur que son interlocuteur, remarquable d'honnêteté intellectuelle et de retenue dans l'expression. Il répond point par point, méthodiquement et fermement. On est loin du $\mathrm{KO}$ : Boulard est debout. Plus que la défense de son livre, il revendique un statut pour la sociologie religieuse : l'expertise, au sens d'une aide à la décision. Ce n'est d'ailleurs pas le dernier article de Boulard sur la question des enquêtes (Cuchet, 2013 : 109-110). La reconnaissance de son travail a lieu à partir des années 1970 par les historiens (plus que par les sociologues), spécialistes du fait religieux ou non ${ }^{11}$.

À l'inverse, Émile Poulat ne consacrera plus d'étude spécifique sur ce sujet après 1970. Il faut dire que cette tradition d'enquêtes sur la pratique dominicale s'éteint en France à cette date-là : 1969 en Haute-Normandie et à Nevers, 1970 à Saint-Nazaire (Chenu, 2011 : 211). La recension du livre de Rémy et Boulard représente l'aboutissement d'une réflexion sur l'historiographie des recensements religieux. Car Poulat, on aura essayé de le montrer, a écrit l'essentiel de ce qu'il pense de la question avant ce débat. Par ses propres recherches sur l'histoire des statistiques religieuses en France, puis en se forgeant une expérience de lecture par le Bulletin, enfin en consacrant deux articles au phénomène de l'urbanisation, il a affuté ses arguments, quitte à reprendre en partie des paragraphes entiers déjà publiés. Il y a surtout cet article de 1969 publié dans Social Compass, qui dit tout, ou presque, de ce que l'auteur expose un an après : le formidable passeur qu'est Boulard qui initie tout un clergé français à une sociologie religieuse en délicatesse à Rome; la faiblesse de cette acculturation malgré tout, car les enquêtes gardent les stigmates d'incompétences et réduisent la sociologie à une sociographie dans l'esprit de nombreux prêtres; les confusions et malentendus qui en résultent sur les rapports entre sociologie et pastorale ; la différence d'exigences internes enfin, entre modes de pensée théologique et démarche scientifique. La sociologie: une discipline pour temps de crise. Sur ce constat de Joachim Wach, Émile Poulat et le chanoine Boulard de 1970 auraient sans doute été d'accord.

Olivier CHATELAN

Laboratoire de recherche historique Rhône-Alpes (LARHRA UMR 5190)

Université Jean Moulin - Lyon 3

olivier.chatelan@univ-lyon3.fr

11. Ainsi François Furet salue en Fernand Boulard « un inventeur de problèmes, ce qui est la manière classique de faire avancer le savoir " dans l'avant-propos du premier volume des Matériaux pour l'histoire religieuse du peuple français XIXe-XXe siècles (cf. Boulard, 1982). 


\section{Bibliographie}

BOULARD Fernard, 1954, Premiers itinéraires en sociologie religieuse, Paris, Éditions Ouvrières.

- 1982, Matériaux pour l'histoire du peuple français XIXe-XX siècles, t. I, Région de Paris, Haute-Normandie, Pays de Loire, Centre, Paris, Presses de la Fondation nationale des sciences politiques-Éditions de l'École des hautes études-Éditions du Centre national de la Recherche scientifique.

Boulard Fernand, RÉMY Jean, 1968, Pratique religieuse urbaine et régions culturelles, Paris, Éditions ouvrières.

- 1970, "Villes et régions culturelles. Acquis et débats ", Archives de sociologie des religions, 29, p. 117-140.

CHenu Alain, 2011, "Les enquêteurs du dimanche. Revisiter les statistiques françaises de pratique du catholicisme (1930-1980) ", Histoire \& Mesure, XXVI-2, p. 177-221.

CuCHET Guillaume, 2013, Faire de l'histoire religieuse dans une société sortie de la religion, Paris, Publications de la Sorbonne, coll. «Itinéraires ».

Foullloux Étienne, et coll., 1997, Les chrétiens français entre crise et libération, $1937-$ 1946, Paris, Éditions du Seuil.

Groupe De Sociologie des Religions, 1969 (a), « Recherches », Archives de sociologie des religions, 28, p. 20-42.

- 1969 (b), «Procédures ", Archives de sociologie des religions, 28, p. 43-56.

Hervieu-Léger Danièle, 2001, "Le catholicisme comme culture ", in Zuber V. (éd.), Émile Poulat. Un objet de science, le catholicisme, Paris, Bayard, p. 85-93.

Labbens Jean, Daille Roger, 1957, La pratique dominicale dans l'agglomération lyonnaise. III. L'instruction, la ville et les pratiquants, Lyon, Institut de sociologie.

LANGLOIS Claude, 2004, "Le catholicisme à la rencontre de la ville, entre après-guerre et Concile ", Les Annales de la recherche urbaine, 96, p. 17-23.

LE BRAs Gabriel, 1931, "Statistique et histoire religieuses ", Revue d'histoire de l'Église de France, 17, p. 425-449.

-, 1956, "Mesures des appartenances religieuses en France. Premier bilan méthodologique ", Archives de sociologie des religions, 2, p. 17-21.

MaÎTRe Jacques, 1957, "Les dénombrements de catholiques pratiquants ", Archives de sociologie des religions, 3, p. 72-95.

-, 2001, "De la première enfance du GSR à l'avenir de la sociologie des religions ", in Zuber V. (dir.), Un objet de science, le catholicisme, Paris, Bayard, p. 25-32.

PIN Émile, 1956, Pratique religieuse et classes sociales dans une paroisse urbaine, SaintPothin à Lyon, Paris, Spes.

Poulat Émile, 1956a, «E. Diebold, La pratique dominicale. Carte religieuse du diocèse de Nice, Nice, Grand séminaire, 1955 », Archives de sociologie des religions, "Bulletin bibliographique ", 1, p. 200.

-, 1956b, "Les cultes dans les statistiques officielles en France ", Archives de sociologie des religions, 2, p. 22-26.

-, 1957a, « Yvan Daniel, L'équipement paroissial d'un diocèse urbain : Paris (1802-1956). Éléments statistiques, Paris, Éditions ouvrières/Économie et Humanisme, 1957 », Archives de sociologie des religions, "Bulletin bibliographique », 3, p. 172-173. 
-, 1957b, "Michel Faerman, Quelques aspects de la pratique religieuse à Mont SaintGuibert, Bruxelles, Institut de sociologie Solvay, 1956 ", Archives de sociologie des religions, "Bulletin bibliographique ", 3, p. 180.

-, 1957c, "Paul Winninger, Construire des églises. Les dimensions des paroisses et les contradictions de l'apostolat dans les villes, Paris, Éditions du Cerf, 1957 », Archives de sociologie des religions, "Bulletin bibliographique ", 4, p. 206-207.

-, 1957d, «La sociologie religieuse et son objet », Critique, 118, mars, p. 229-242.

-, 1958a, "Une enquête anticléricale de pratique religieuse en Seine-et-Marne (1903) ", Archives de sociologie des religions, 6, p. 127-148.

-, 1958b, « Jesus-Maria Vasquez, Asi viven y mueren, Pampelune, Éditorial OPE, 1958 ", Archives de sociologie des religions, "Bulletin bibliographique ", 7, p. 188.

-, 1960a, "Socialisme et anticléricalisme. Une enquête socialiste internationale (19021903) ", Archives de sociologie des religions, 10, p. 109-131.

-, 1960b, "La découverte de la ville par le catholicisme français contemporain ", Annales, 16-6, p. 1168-1179.

-, 1961, "Les nouveaux espaces urbains du catholicisme français ", Cahiers internationaux de sociologie, 30, p. 115-129.

-, 1967, "Joseph Laloux, Manuel d'initiation à la sociologie religieuse, Paris, Éditions universitaires, 1967 ", Archives de sociologie des religions, "Bulletin bibliographique ", 24, p. 210.

-, 1969, "Trois problèmes pour la sociologie du catholicisme ", Social Compass, 4, p. 471-483.

-, 1970, "Catholicisme urbain et pratique religieuse ", Archives de sociologie des religions, 29, p. 97-116.

SORrel Christian, 2013, "Le chanoine Boulard, un inconnu célèbre ", in Id. (éd.), Les "Matériaux Boulard "trente ans après, Chrétiens et sociétés, Lyon, RESEA-LARHRA, coll. « Documents et Mémoires », p. 9-36. 